\title{
ANÁLISIS DE LA VARIABILIDAD ESPACIO- TEMPORAL DE LAS PRECIPITACIONES EN EL SECTOR ESPAÑOL DE LA CUENCA DEL DUERO (1961-2005)
}

\author{
Antonio Ceballos Barbancho \\ Departamento de Geografía, Universidad de Salamanca \\ ceballos@usal.es \\ Enrique Morán Tejeda \\ Juan Ignacio López Moreno \\ Instituto Pirenaico de Ecología, CSIC (Zaragoza)
}

\section{RESUMEN}

En este trabajo se estudia la variabilidad espacio-temporal y la tendencia de las precipitaciones (1961-2005, n=45) en el sector español de la cuenca del Duero mediante el análisis de series homogéneas de datos mensuales y anuales correspondientes a un total de 214 estaciones. Los resultados obtenidos muestran una acusada variabilidad estacional, interanual y espacial de las precipitaciones, lo que impide la observación de tendencias estadísticamente significativas, tanto en las series mensuales como anuales. Sólo en el mes de febrero se detectó una tendencia negativa significativa en gran parte del territorio de la cuenca. Con algunos matices, los resultados del análisis confirman la ausencia de tendencias claras observadas en Europa meridional durante la segunda mitad del siglo XX y primeros años del siglo XXI.

Palabras clave: precipitaciones, variabilidad espacio-temporal, tendencia temporal, cuenca del Duero.

Fecha de recepción: junio 2011.

Fecha de aceptación: diciembre 2012. 


\section{ABSTRACT}

This work focuses on the spatial and temporal variability of precipitation in the Spanish sector of the Duero basin, for the period 1961-2005 $(n=45)$, and the analysis of temporal trends. Results highlight a marked variability on the seasonal, inter-annual and spatial basis, which usually mask the existence of trends at the monthly and annual scale. Thus, only February shows a negative and significant trend across most of the territory, while annual precipitation has remained rather stationary at the long-term. These results confirm, with few exceptions, the absence of significant trends of precipitation in Southern Europe during the second half of the 20th century.

Key words: precipitation, spatial and temporal variability, temporal trends, Duero basin.

\section{INTRODUCCIÓN}

Históricamente las precipitaciones en los países mediterráneos se han caracterizado por su variabilidad natural, tanto estacional como interanual. En un contexto general de «cambio climático», en donde el incremento inequívoco de las temperaturas contrasta con la multidireccionalidad de las precipitaciones, es lógico pensar que el aumento de energía en el sistema atmosférico, inducido por el calentamiento antrópico, repercuta en un incremento de esta variabilidad con la ocurrencia de precipitaciones más intensas e irregulares. A modo de ejemplo, en los últimos años en España se han sucedido años muy secos (2005) y muy húmedos (2010), con los problemas de abastecimiento de demanda hídrica o desbordamiento de cauces e inundaciones según el cariz de cada situación. El comportamiento interanual de las precipitaciones, sus tendencias, su variabilidad pasada y futura, así como las diferencias espaciales de estos cambios, representan algunos de los procesos más estudiados por las ciencias del clima. Las principales razones son su inherente naturaleza caótica, su importancia para la vida y el desarrollo de las sociedades, el efecto que sobre ellas puede tener el calentamiento atmosférico o su indudable relación con el ciclo hidrológico.

Dentro de estos estudios estarían, por un lado, aquellos encaminados a predecir las tendencias futuras, a corto y medio plazo, de las principales variables climáticas. En el caso de las precipitaciones, a escala global, el Intergovernmental Panel on Climate Change -IPCC(2007) prevé para finales del siglo XXI un aumento del desequilibrio actual del reparto de las precipitaciones, con un descenso de las mismas entre $\operatorname{los} 5^{\circ}$ y $45^{\circ}$ de latitud de ambos hemisferios y un incremento en el resto del planeta. A escala regional García-Ruiz et al. (2011), para el conjunto de la cuenca mediterránea, pronostican una reducción de las precipitaciones entre un 6 y un 14\% respecto a la media del último período climático normal (1961-90), mientras que en el caso de España Ayala-Carcedo e Iglesias (2000) esperan una reducción media de las precipitaciones del $8 \%$, que en el caso concreto de la cuenca del Duero sería del $7 \%$.

Un segundo grupo de trabajos, entre los que se incluye el presente, tienen por objeto describir y analizar los cambios ocurridos en las últimas décadas para conocer mejor el funcionamiento del sistema climático y sus consecuencias sobre diversos sistemas naturales 
y socio-económicos. En esta línea, los estudios regionales realizados en nuestro entorno geográfico próximo muestran el contraste entre el aumento de las precipitaciones observado durante el siglo XX en la Europa septentrional frente a la mayor incertidumbre de los países de la Europa mediterránea, en donde, o bien no se ha observado tendencia alguna, o bien estas han sido negativas, pero casi siempre no significativas aplicando los tests estadísticos al uso (Zhang et al. 2007; Bladé y Castro-Diez, 2010). Como anteriormente comentamos, la enorme variabilidad espacio-temporal de las precipitaciones demanda la realización de estudios a gran escala que permitan conocer con cierto grado de detalle su patrón de comportamiento y compararlo con el de otros territorios, con el objeto de comprobar si es posible o no una agregación espacial de las tendencias observadas. El desarrollo de este tipo de análisis en el ámbito territorial de una gran cuenca hidrográfica posibilita, con carácter aplicado, estudiar la relación entre precipitaciones y caudales y evaluar la evolución de los recursos hídricos disponibles.

En el caso de la cuenca del Duero existen dos precedentes de estudios generales sobre la dinámica de las precipitaciones: por un lado el trabajo realizado por Del Río et al. (2005), quienes analizaron los valores medios tanto mensuales como anuales de 171 estaciones pluviométricas para el período 1961-1997, y por otro el publicado por Labajo y Piorno (2001), quienes también se ocuparon de la evolución temporal de las precipitaciones en Castilla y León durante el período 1931-1996, a partir de una serie pluviométrica homogeneizada con los datos de tan sólo 44 estaciones.

Considerando estos antecedentes, los objetivos principales del análisis que presentamos son los siguientes:

i) Analizar la tendencia temporal de las precipitaciones anuales y mensuales en el sector español de la cuenca del Duero para el período 1961-2005, evaluando el significado estadístico de las mismas. Con ello integramos los años que conforman el último período climático normal (1961-90) y los correspondientes a la última década del siglo XX y comienzo del siglo XXI, momento en el cual han sido más evidentes las anomalías climáticas respecto al citado período de referencia.

ii) Identificar los patrones espaciales de los cambios, mediante la interpolación espacial de las precipitaciones, a partir de los datos puntuales de los observatorios seleccionados.

iii) Comparar y discutir las diferencias y similitudes del patrón espacio-temporal de precipitaciones en la cuenca del Duero con el encontrado por otros autores en un entorno geográfico próximo (Península Ibérica).

\section{II. ÁREA DE ESTUDIO}

La cuenca del Duero se encuentra situada en la mitad norte de la Península Ibérica entre $\operatorname{los} 40^{\circ} 15^{\prime}$ y $43^{\circ} 07^{\prime}$ de latitud norte y los $2^{\circ} 09^{\prime}$ y $8^{\circ} 40^{\prime}$ de longitud oeste. Constituye uno de los sistemas hidrográficos de mayor entidad de la Península Ibérica, con una superficie total de $97.290 \mathrm{~km}^{2}$, de los cuales la mayor parte $\left(78.952 \mathrm{~km}^{2}\right)$ pertenecen a España (figura 1).

Desde un punto de vista fisiográfico, el sector español de la cuenca del Duero está formado por una gran llanura elevada (Submeseta Norte) rodeada por una orla montañosa. La llanura, no exenta de irregularidades topográficas como consecuencia de la actividad fluvial 


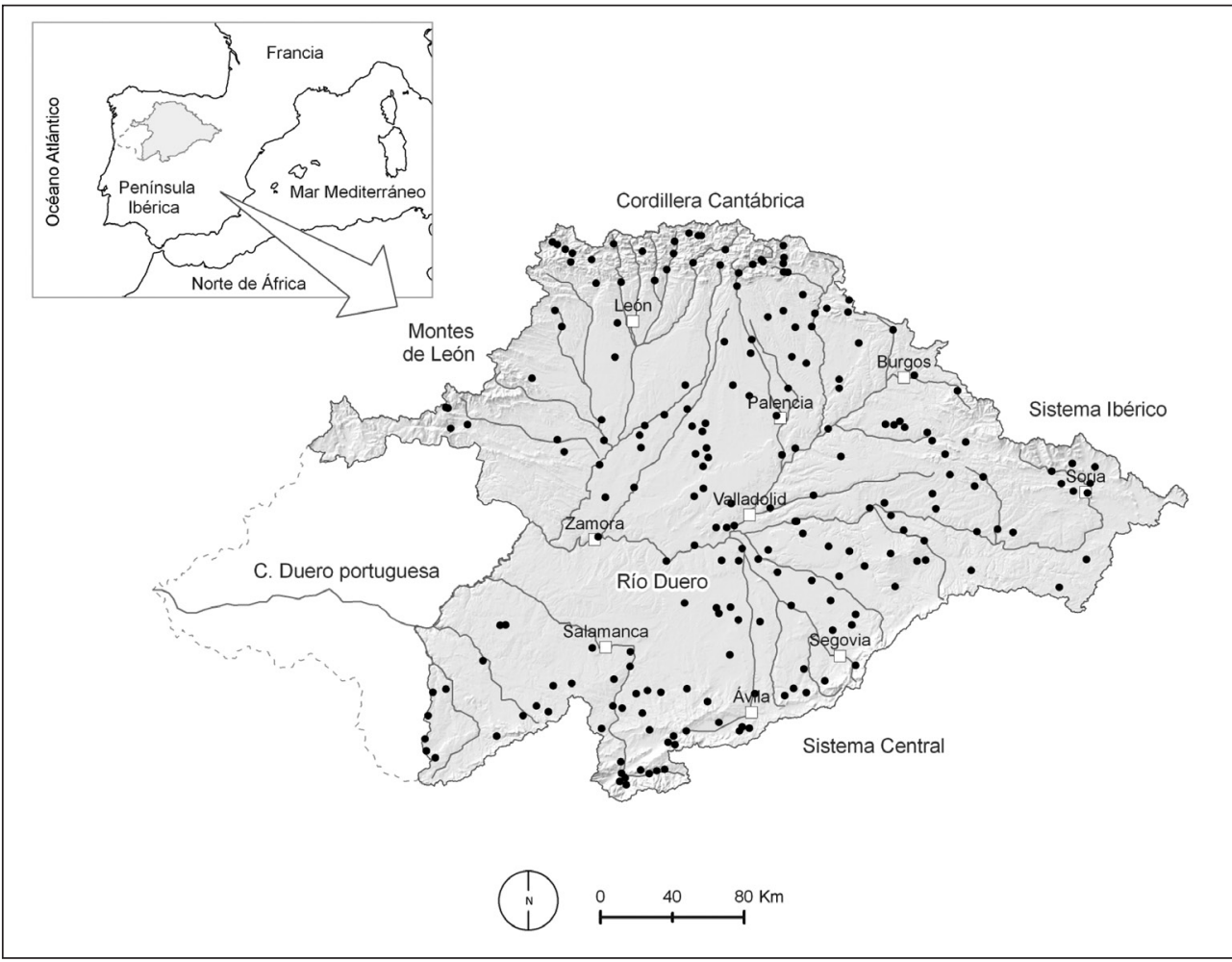

desde finales del Terciario, está a su vez compuesta por dos unidades bien diferenciadas por sus características estructurales y composición litológica: la penillanura del sector occidental sobre materiales del Paleozoico y la planicie de sedimentos del Cenozoico, que se extiende por los sectores central y oriental de la cuenca, con plataformas estructurales horizontales delimitadas por abruptos escarpes y extensas campiñas. El borde montañoso tampoco es homogéneo, presentando una serie de unidades distintas atendiendo a su estructura y composición litológica: Montes de León o Montañas Galaico-Leonesas, que representan el límite noroccidental de la cuenca, formadas por materiales paleozoicos y con altitudes superiores a los $2.000 \mathrm{~m}$. El límite norte lo forma la Cordillera Cantábrica, con cumbres próximas a los $2.500 \mathrm{~m}$. y una estructura geológica compleja en cuanto a su origen, con materiales diversos del Paleozoico y Cenozoico. El Sistema Ibérico delimita la parte oriental de la cuenca y está formado por materiales mesozoicos de origen sedimentario diverso, con cimas que superan los $2.000 \mathrm{~m}$. Finalmente, por el sur la cuenca está delimitada por el Sistema Central con materiales paleozoicos, fracturados y deformados por la orogenia alpina, y cumbres que alcanzan los 2.500 m (Cabo y Manero, 1990, Fernández, 1992, Jiménez, 1997). 
El régimen térmico de la cuenca es el característico de un clima de interior, con una apreciable oscilación térmica entre los meses de invierno, con temperaturas medias inferiores a $\operatorname{los} 5^{\circ} \mathrm{C}$, y los meses de verano, con medias que pueden superar $\operatorname{los} 20^{\circ} \mathrm{C}$. En cuanto a su distribución espacial, las temperaturas más altas se registran en el sector suroccidental de la cuenca, con medias anuales superiores a $\operatorname{los} 15^{\circ} \mathrm{C}$, mientras que en las cumbres de la Cordillera Cantábrica y del Sistema Central las medias anuales no superan los $5^{\circ} \mathrm{C}$. La mayor parte del territorio se encuentra entre las isotermas de $\operatorname{los} 10^{\circ}$ y $12^{\circ} \mathrm{C}$ de media anual. Por su parte, el régimen pluviométrico es la característica que define la mediterraneidad del clima, ya que se registra un período húmedo entre el otoño y la primavera y un período con escasa pluviosidad en los meses estivales. Junto a la estacionalidad, una segunda característica de las precipitaciones en la cuenca es la marcada disimetría pluviométrica entre el interior (en donde en algunas zonas no se sobrepasan los $400 \mathrm{~mm}$ anuales) y el borde montañoso con más de $1.000 \mathrm{~mm}$ anuales. A pesar del predominio de un clima mediterráneo continentalizado en la mayor parte de la cuenca, en los rebordes montañosos sería más apropiado hablar de climas tanto sub-mediterráneos como sub-atlánticos (García Fernández, 1986, Cabo y Manero, 1990).

Como consecuencia de la configuración fisiográfica y de las características del clima, pasado y actual, en la cuenca se ha articulado una red hidrográfica extensa y compleja con el río Duero como principal colector y una aportación media anual en el sector español próxima a los $13.800 \mathrm{hm}^{3}$. Las montañas que rodean la meseta son la fuente principal de los recursos hídricos, al actuar estas de reservorio de las precipitaciones y alimentar directamente a los ríos afluentes más caudalosos como el Esla (5.040 hm³/año) o el Pisuerga $\left(2.400 \mathrm{hm}^{3} /\right.$ año$)$. Junto a los recursos hídricos superficiales habría que subrayar la presencia de importantes formaciones acuíferas, que pueden acumular anualmente unos $1.500 \mathrm{hm}^{3}$ (Cabo y Manero, 1990, Fernández, 1992, www.chduero.es, www.hercules.cedex.es).

Finalmente, en relación con los usos del suelo y distribución de las formaciones vegetales, destaca el contraste existente entre el paisaje de la llanura interior sedimentaria y el de la penillanura occidental y los rebordes montañosos de la cuenca. La mayor parte del territorio de la llanura interior sedimentaria está ocupado por terrenos de cultivo dedicados al cereal, viñedo y regadío. En esta unidad las formaciones forestales se restringen a algunas superficies de coníferas de cierta entidad (e.g. Tierra de Pinares), principalmente con pino negral (Pinus pinaster) y piñonero ( $P$. pinea), repoblaciones localizadas de pino carrasco (P. halepensis), la presencia generalizada de rebollos o melojos (Quercus pyrenaica) sobre los sedimentos silíceos, manchas de encinas ( $Q$. ilex sp. ballota) y quejigos $(Q$. faginea sp. faginea) dispersas, enebros (Juniperus oxycedrus) y sabinas (J. thurifera) en los páramos cálcáreos, y de bosquetes riparios de chopos (Populus nigra y P. alba), sauces (Salix sp.), alisos (Alnus glutinosa) y fresnos (Fraxinus sp.). Las formaciones netamente forestales están más extendidas por las penillanuras occidentales, con encinas ( $Q$. ilex $\mathrm{sp}$. ballota), melojos ( $Q$. pyrenaica) y quejigos ( $Q$. faginea sp. faginea) y en los sistemas montañosos que delimitan la cuenca, con un marcado contraste bioclimático que explica la presencia de abedulares (Betula pendula), hayedos (Fagus sylvatica), robledales ( $Q$. petraea) y pinares (P. sylvestris) en las montañas septentrionales, y de melojares $(Q$. pyrenaica) y pinares $(P$. pinaster, $P$. nigra y $P$. sylvestris) en las montañas orientales y meridionales (Ruiz de la Torre, 1996). 


\section{MATERIAL Y MÉTODOS}

\section{Elaboración de una base de datos pluviométricos homogénea}

Para la realización del presente análisis se utilizaron series mensuales y anuales de precipitaciones procedentes de la red de estaciones pluviométricas de la Agencia Estatal de Meteorología (AEMET), abarcando el período 1961-2005 (n=45). La selección del período de estudio respondió a las siguientes premisas: i) que la serie de datos tuviera una duración superior a 30 años, de acuerdo con las recomendaciones de la Organización Meteorológica Mundial para definir y estudiar la variabilidad de un clima concreto, ii) que las series incluyeran el último periodo climático de referencia (1961-90), utilizado por el IPCC para el estudio de las anomalías climáticas observadas durante las últimas décadas, y iii) que las series recogieran los últimos años del siglo XX y comienzos del siglo XXI, en principio los más sensibles a la ocurrencia de un hipotético «cambio climático» inducido por actividades antrópicas.

Una vez definido el período de estudio, muchas series de datos disponibles fueron descartadas para el análisis por su reducida extensión y el resto fueron testadas atendiendo a una serie de criterios de control de calidad (Peterson et al., 1998) como existencia de valores extremos (outliers), de inhomogeneidades (cambios bruscos en la tendencia de una variable) o ausencia de datos puntuales en las series (lagunas) debidos a cambios en el instrumental de medición, reubicación del mismo, errores en la medición y toma de datos, paradas temporales en su actividad, etc.

Para la eliminación de los valores extremos (outliers) se identificaron los datos de cada mes (con la excepción de julio y agosto debido al carácter localizado e intenso de las precipitaciones convectivas estivales) que mostraron un valor de precipitación superior a 4 veces la desviación estándar respecto a los tres observatorios más cercanos y con datos disponibles para ese mes. Estos datos se etiquetaron para comprobar su grado de coincidencia con los observatorios cercanos. En el caso de que se tratara de un dato extremo se decidió eliminarlo y no incluirlo en el análisis.

Para evaluar la homogeneidad de las series, se construyeron series de referencia de acuerdo con la propuesta de Peterson y Easterling (1994). Siguiendo las recomendaciones de los citados autores, en primer lugar se elaboró una matriz de correlaciones entre las series y se seleccionaron las 5 series que mayor coeficiente de correlación mostraron con cada una. Posteriormente se calculó la media de las precipitaciones de las 5 series, ponderada según el cuadrado del coeficiente de correlación:

$$
P_{R, i}=\frac{\left(P_{R 1, i} \cdot R_{1}^{2}\right)+\left(P_{R 2, i} \cdot R_{2}^{2}\right)+\left(P_{R 3, i} \cdot R_{3}^{2}\right)+\left(P_{R 4, i} \cdot R_{4}^{2}\right)+\left(P_{R 5,1} \cdot R_{5}^{2}\right)}{R_{1}^{2}+R_{2}^{2}+R_{3}^{2}+R_{4}^{2}+R_{5}^{2}}
$$

donde $P_{R, i}$ es la precipitación de la serie de referencia en el mes $i, P_{R x, i}$ es el valor de precipitación de la estación $x$ en el mes $i$ y $R_{i}$ es el coeficiente de correlación entre la serie de la estación candidata y la estación de referencia. De este modo se obtuvieron 296 series de referencia que se utilizaron para testar la homogeneidad de las series originales. De entre los distintos métodos de homogenización existentes se optó por utilizar el denominado Standard 
Normal Homogeneity Test -SNHT- (Alexandersson, 1986) por su contrastada aceptación y utilización en la homogeneización de variables climáticas (e.g. Keiser y Griffiths, 1997, Moberg y Bergstrom, 1997, De Luis et al., 2009 y Vicente-Serrano et al., 2009). En el caso de las precipitaciones, este método se basa en la creación de una serie de ratios entre la serie candidata y la serie de referencia para detectar las inhomogeneidades, consideradas como rupturas en las medias entre distintos periodos.

Finalmente, para el relleno de datos ausentes de las series, el punto de partida fue considerar sólo aquellos observatorios que tuvieran menos del $15 \%$ de los datos mensuales ausentes durante el período de estudio (1961-2005). Una vez hecha la selección, para la supresión de lagunas se optó por la aplicación de un ajuste lineal entre la serie con lagunas (candidata) y su serie de referencia (predictora), de acuerdo con la propuesta de Peterson y Easterling (1994). En los casos en los que los valores de la serie candidata se ajustaron al modelo, con un coeficiente de correlación superior a $0,7(R>0,7)$ significativo al $95 \%$ (valor-p $<0,05)$, se calcularon los valores ausentes de la serie candidata aplicando la ecuación resultante.

Con este filtro se obtuvieron series homogéneas de datos pluviométricos, en las que se asume que las variaciones se han producido exclusivamente por causas naturales (Easterling y Peterson, 1995) y que son, por lo tanto, aptas para realizar cualquier test o procedimiento estadístico (Lanzante, 1996). De las 1.500 estaciones pluviométricas de la AEMET que registran (o han registrado) datos en el territorio de Castilla y León finalmente fueron seleccionadas 214. Aunque el número de estaciones se redujo muy notablemente, aún así resultó suficiente para representar la variabilidad espacial y temporal. La figura 1 muestra la localización de las estaciones pluviométricas seleccionadas, apreciándose un cierto contraste entre la densidad de estaciones situadas en las zonas de montaña y aquellas ubicadas en determinadas áreas del interior, lo que teóricamente no debería representar ningún tipo de problema ya que, con la excepción de los episodios convectivos estivales, las precipitaciones que ocurren en el interior de la cuenca suelen estar asociadas a sistemas frontales atlánticos que barren el territorio interior llano de forma homogénea con una menor variabilidad espacial que en las zonas de montaña.

\section{Interpolación espacial de los datos pluviométricos}

Para estimar la tendencia temporal de las precipitaciones en el conjunto de la cuenca e identificar los patrones espaciales del cambio se procedió a una interpolación espacial de las precipitaciones a partir de los datos puntuales de los observatorios seleccionados. De acuerdo con la propuesta de Ninyerola et al. (2000) se construyeron modelos de regresión múltiple con variables independientes (en nuestro caso altitud, latitud y longitud), que presentaron una distribución espacial continua (capas ráster) y podían explicar la distribución espacial de la variable dependiente (precipitaciones). Con la implementación de un sistema de información geográfica (ArcGIS) se obtuvieron los modelos digitales del terreno (MDT) de las tres variables independientes con un tamaño de celda de 100 x 100 m., calculándose así el valor de altitud, latitud y longitud en cada píxel de la cuenca. Con los valores mensuales de precipitación (promedios del período de estudio), altitud, latitud y longitud, en cada observatorio, se calcularon las regresiones múltiples para los doce meses del año según la siguiente ecuación: 


$$
y=b_{0}+b_{1}(\text { lat })+b_{2}(\text { long })+b_{3}(\text { alt })
$$

donde $b_{n}$ son los coeficientes de la regresión múltiple ajustados para cada mes y variable dependiente (precipitación). El método utilizado para construir el modelo es el conocido como regresión estadística o regresión «paso a paso», y se basa en criterios puramente estadísticos para introducir las variables independientes (Tabachnick \& Fidell 1996). En este caso, las variables fueron introducidas en el modelo tan sólo si presentaban una contribución estadísticamente significativa $(\alpha<0,05)$ a la explicación de la variable dependiente, descartándose si no cumplían con dicho criterio.

Con la aplicación del modelo se obtuvieron los valores de precipitación para cada mes y píxel de 100 x 100 m. en toda la superficie de la cuenca, pudiéndose entonces generar los mapas de distribución de las precipitaciones en el sector español de la cuenca del Duero. Estos mapas no se consideraron definitivos ya que fueron refinados comprobando la bondad de ajuste del modelo. Para ello se calcularon los errores residuales en cada punto real del muestreo (observatorios) mediante:

$$
\text { Residual }=\text { Valor }_{\text {observado }}-\text { Valor }_{\text {predicho }}
$$

Con ello se asume que sumando los residuales a los valores predichos refinaríamos los resultados obteniendo un segundo mapa más real que el primero.

\section{Cálculo de tendencias}

Para detectar tendencias en las series de datos y su significación estadística, los tests no paramétricos son recomendados frente otras pruebas más clásicas (Pearson, t-test), ya que se basan en los rangos de los registros y no en el valor de los mismos. Por ello, son independientes de los valores y de la distribución de las variables analizadas y no requieren asumir la normalidad de las muestras. Los tests no paramétricos más utilizados son el test de rangos de Spearman (Sneyers, 1990) y el test de Mann-Kendall (Mann, 1945, Kendall, 1975), cuya validez y robustez ha sido suficiente contrastada con resultados similares (e.g. Hirsch et al., 1982, Berryman et al., 1988, Sneyers, 1992, Yue et al., 2002). En el presente trabajo aplicamos el test Mann-Kendall, tras comprobar su similitud con los resultados obtenidos del test de Spearman, por su mayor grado de utilización en el análisis de variables climáticas e hidrológicas.

El test se aplicó mediante el paquete estadístico SPSS ${ }^{\circ}$, basándose en un algoritmo de correlación entre la variable X (años) y la variable Y (precipitaciones). Las observaciones se ordenaron separadamente de forma ascendente y fueron reemplazadas por sus rangos. La aplicación del test de Mann-Kendall nos devuelve dos parámetros que sirven para constatar la existencia, o no, de tendencias en las series (estadístico tau $-\boldsymbol{\tau}-$ ) y su grado de significación estadística $(\alpha)$. Un $\tau=0$ indica la inexistencia de tendencia, mientras que conforme se aleje de 0 y se aproxime a 1 estaríamos ante la existencia de una tendencia bien positiva o negativa dependiendo del signo de la misma. En el caso de $\alpha$, en el presente trabajo hemos considerado un valor $\alpha<0,05$ para determinar una tendencia como estadísticamente significativa al suponer una probabilidad de certeza del $95 \%$ de que la misma no sea causada por un muestreo aleatorio. 


\section{RESULTADOS}

\section{Distribución espacial de las precipitaciones y régimen pluviométrico}

La precipitación de la cuenca del Duero muestra una gran variabilidad tanto espacial como estacional. Como se aprecia en la figura 2, la distribución de la precipitación anual en la cuenca está estrechamente ligada con la topografía, correspondiendo las zonas más pluviosas $(>2.000 \mathrm{~mm})$ a los rebordes montañosos y las de menor cuantía $(<500 \mathrm{~mm})$ al interior, observándose por tanto un marcado gradiente pluviométrico dentro de la cuenca desde las condiciones hiperhúmedas en la zona periférica a las semiáridas del interior continental, debido a su aislamiento de las masas de aire húmedo del Atlántico. Este gradiente noroestesudeste es especialmente manifiesto en el semestre más húmedo del año (octubre-marzo) en relación con la frecuencia de entradas de masas de aire húmedo del Atlántico en la Península Ibérica, que son la principal fuente de precipitación durante el otoño y el invierno (figura 3).

La tabla 1 muestra los coeficientes y el grado de significación estadística de las variables independientes introducidas en las regresiones lineales para interpolar espacialmente la precipitación en la cuenca. El efecto de la topografía sobre la precipitación queda constatado en

Figura 2

DISTRIBUCIÓN DE LA PRECIPITACIÓN ANUAL EN LA CUENCA DEL DUERO

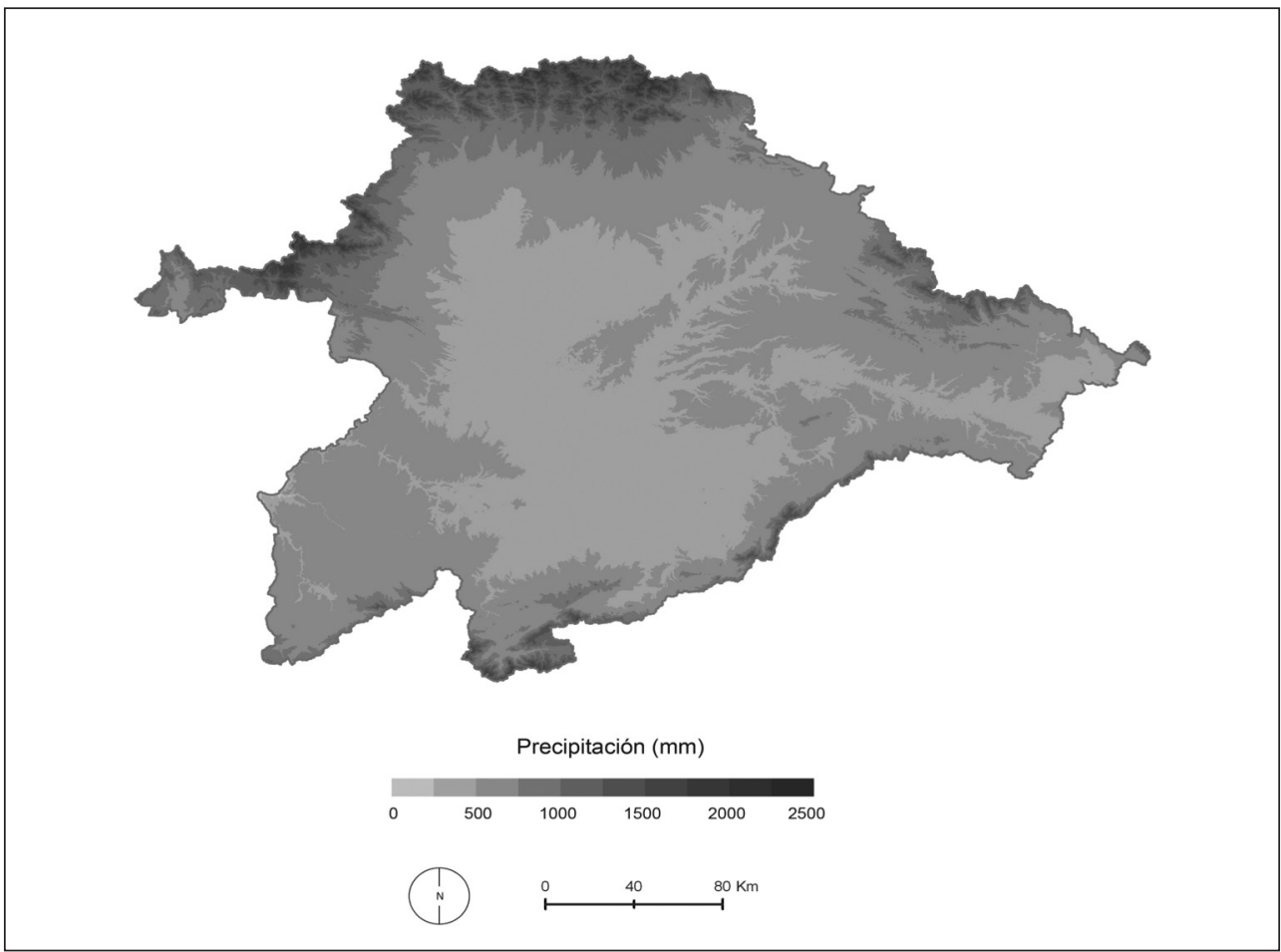

Fuente: Elaboración propia a partir del análisis de 214 estaciones pluviométricas. 
Figura 3

DISTRIBUCIÓN ESPACIAL DE LA PRECIPITACIÓN EN LA CUENCA DEL DUERO CORRESPONDIENTE A CADA UNO DE LOS 12 MESES DEL AÑO
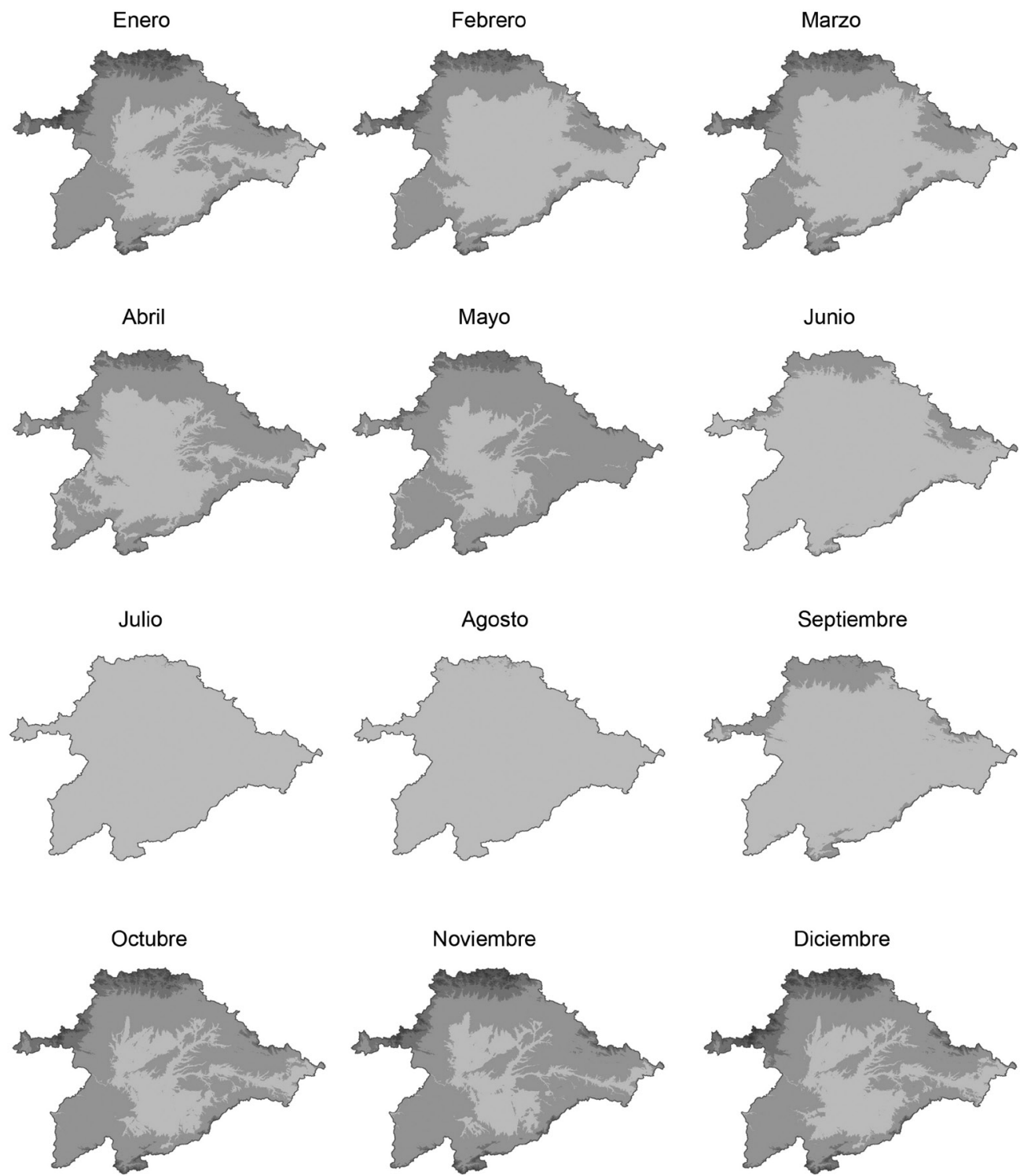

Precipitación (mm)

$50 \quad 100 \quad 150 \quad 200 \quad 250 \quad 300$ 
el hecho de que la principal variable que explica la distribución de las precipitaciones sea la altitud, con la excepción de los meses de julio y agosto debido a la ocurrencia de lluvias convectivas aleatoriamente distribuidas en el espacio.

A partir de los coeficientes mostrados en la tabla 1 se realizaron las interpolaciones para describir la distribución de la precipitación anual y mensual en la cuenca (figuras 2 y 3 ). En la figura 4, elaborada con las series de precipitaciones mensuales de las 214 estaciones analizadas, se aprecia que tras el mínimo pluviométrico estival propio de los territorios mediterráneos, a partir de octubre comienza la estación lluviosa que se prolonga en los meses de noviembre, diciembre y enero. Entre febrero y abril se produce un descenso tras el cual ocurre un repunte en mayo. Este régimen medio se corresponde en líneas generales con el patrón habitual de distribución intraanual de las precipitaciones en el oeste peninsular y está directamente controlado por la circulación de vientos del oeste y la ubicación y potencia del Anticiclón de las Azores (Trigo y Palutikof, 2001, Paredes et al., 2006). El rango intercuartil ilustra la mayor variabilidad espacial de las precipitaciones en aquellos meses en los que estas son más elevadas, debido al marcado gradiente entre los rebordes montañosos y el interior de la cuenca en otoño e invierno, mientras que en los meses de primavera y verano las precipitaciones son de menor cuantía y espacialmente más homogéneas.

Tabla 1

COEFICIENTES DE LAS VARIABLES INDEPENDIENTES UTILIZADAS PARA INTERPOLAR ESPACIALMENTE LAS PRECIPITACIONES DE LA CUENCA Y SU PARTICIPACIÓN (R²) EN EL MODELO. LOS VALORES EN BLANCO (-) INDICAN QUE LAPARTICIPACIÓN DE LA VARIABLE NO ES SIGNIFICATIVAAL 95\% DE CONFIANZA

\begin{tabular}{|c|c|c|c|c|c|c|c|c|}
\hline \multirow{2}{*}{ Mes } & \multirow{2}{*}{ Constante } & \multicolumn{2}{|c|}{ Altitud } & \multicolumn{2}{c|}{ Longitud } & \multicolumn{2}{c|}{ Latitud } & \multirow{2}{*}{ Total $\mathbf{R}^{2}$} \\
\cline { 3 - 8 } & & coef. & $\mathbf{R}^{\mathbf{2}}$ & coef. & $\mathbf{R}^{\mathbf{2}}$ & coeff. & $\mathbf{R}^{\mathbf{2}}$ & \\
\hline Ene & $-701,96$ & 0,109 & 0,38 & $-0,00018$ & 0,14 & 0,00016 & 0,16 & 0,69 \\
\hline Feb & $-556,92$ & 0,095 & 0,41 & $-0,00015$ & 0,15 & 0,00012 & 0,14 & 0,70 \\
\hline Mar & $-503,17$ & 0,094 & 0,50 & $-0,00011$ & 0,13 & 0,00011 & 0,10 & 0,73 \\
\hline Abr & $-444,07$ & 0,074 & 0,45 & $-0,00006$ & 0,06 & 0,00010 & 0,13 & 0,64 \\
\hline May & $-406,04$ & 0,072 & 0,52 & $-0,00006$ & 0,05 & 0,00009 & 0,14 & 0,72 \\
\hline Jun & $-215,08$ & 0,035 & 0,49 & - & - & 0,00005 & 0,18 & 0,67 \\
\hline Jul & $-266,41$ & 0,018 & 0,22 & 0,00001 & 0,01 & 0,00006 & 0,56 & 0,79 \\
\hline Ago & $-280,99$ & 0,024 & 0,31 & 0,00001 & 0,01 & 0,00006 & 0,52 & 0,84 \\
\hline Sep & $-308,11$ & 0,050 & 0,46 & $-0,00007$ & 0,14 & 0,00007 & 0,14 & 0,74 \\
\hline Oct & $-551,73$ & 0,110 & 0,45 & $-0,00017$ & 0,16 & 0,00012 & 0,12 & 0,73 \\
\hline Nov & $-656,03$ & 0,126 & 0,47 & $-0,00016$ & 0,10 & 0,00014 & 0,13 & 0,70 \\
\hline Dic & $-793,78$ & 0,131 & 0,44 & $-0,00020$ & 0,13 & 0,00018 & 0,16 & 0,73 \\
\hline
\end{tabular}


Figura 4

RÉGIMEN MENSUAL DE LAS PRECIPITACIONES EN LA CUENCA DEL DUERO. LA LÍNEA CON ROMBOS CORRESPONDE A LOS VALORES MEDIOS DE LAS 214 ESTACIONES PLUVIOMÉTRICAS Y EL SOMBREADO GRIS MUESTRA EL RANGO ENTRE EL 25\% Y EL 75\% DE LOS CASOS (CUARTILES PRIMERO Y TERCERO)

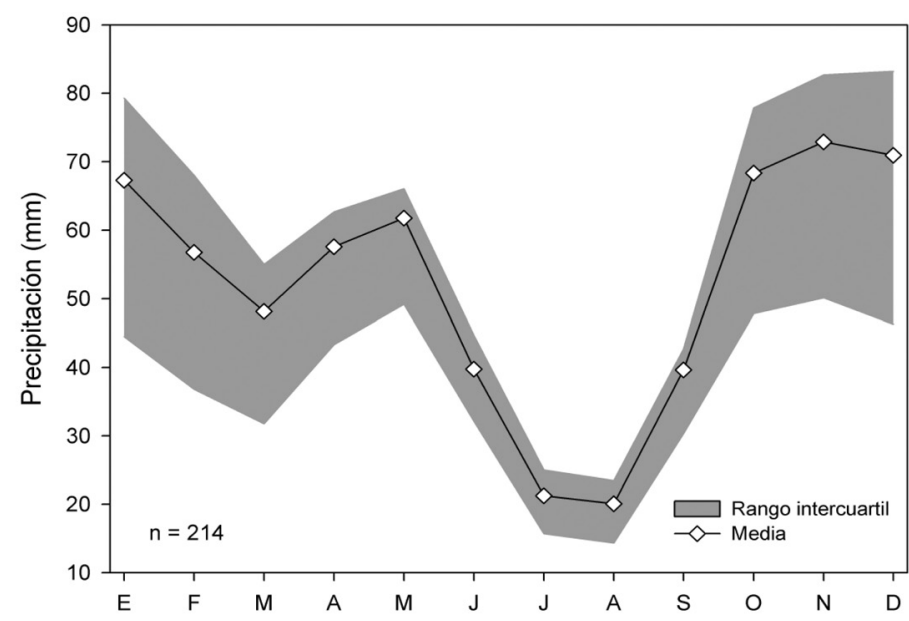

\section{Evolución y tendencia de las precipitaciones mensuales y anuales}

Las figuras 5 y 6 muestran la evolución de las precipitaciones anuales y mensuales del conjunto de la cuenca durante el período de estudio. De manera complementaria, la tabla 2 refleja los estadísticos de dispersión y cambio (incluyendo las tendencias). La primera característica que se observa, tanto en las figuras como en la tabla, es la elevada tasa de variabilidad interanual, con coeficientes de variación superiores al 60\% en la mayoría de los meses, superándose el $100 \%$ en los meses de verano. Respecto a la serie de precipitaciones anuales el coeficiente de variación es igual a 23\%, por encima del umbral del $20 \%$ que separan los ombroclimas mediterráneo y atlántico en la Península Ibérica (De Castro et al., 2005). No obstante, como ilustra la figura 7, el valor medio se ve superado en gran parte de la cuenca, ya que tan sólo el sector noroeste de la misma registra valores por debajo del $20 \%$. De nuevo es evidente el gradiente noroeste-sudeste de la cuenca, con los valores más altos $(>28 \%)$ en las zonas central y suroriental de la misma, siendo por tanto la variabilidad interanual mayor en el territorio con menor pluviometría. Este hecho es un indicador más de las condiciones de mediterraneidad propias de los sectores central y oriental de la cuenca respecto a las condiciones más atlánticas propias del noroeste.

Es precisamente esta variabilidad lo que obstaculiza la posibilidad de observar tendencias significativas en las series. Para intentar poder apreciar algún tipo de ciclo o tendencia en las series se ha aplicado una media móvil con una ventana de 5 años al rango intercuartil de las 214 series. En primer lugar se detecta que no existe un patrón de cambio común entre los meses, ya que cada uno muestra una particular evolución de las precipitaciones. En algunos meses se aprecian ciclos más o menos decadales de subida y bajada de las precipitaciones 
(enero y febrero), mientras que en otros meses (mayo, junio y diciembre) los ciclos son de mayor amplitud o no se vislumbra una evolución cíclica (marzo y septiembre). Tal y como han demostrado Rodríguez Puebla et al. (2001), Visbeck et al. (2001) y Osborn (2004), entre otros, los ciclos cuasi decadales en las precipitaciones de invierno son uno de los patrones de variabilidad de las precipitaciones más comunes en el oeste peninsular y están estrechamente relacionados con la variabilidad propia de la Oscilación del Atlántico Norte. En la tabla 2 aparecen las tendencias en las precipitaciones medias de la cuenca ( $\tau$ de Mann-Kendall) y su significación estadística $(\alpha)$. La mayoría de los meses no muestran tendencias temporales significativas, con la excepción de junio y agosto con unas tendencias negativa y positiva respectivamente. Las precipitaciones de febrero y octubre también han registrado una tendencia negativa y positiva con unos valores que se encuentran en el límite de la significación estadística establecida. Mediante un ajuste lineal se ha estimado el porcentaje de cambio que se ha producido entre el comienzo y el final del período. Para los meses citados anteriormente, las precipitaciones han ascendido en más de un $130 \%$ y $104 \%$ en agosto y octubre respectivamente y se han reducido en un $56 \%$ y un $24 \%$ en febrero y junio. No obstante, la importancia de tales cambios, con independencia de su magnitud, radica en el volumen de las precipitaciones registrado en cada mes. En este caso, los meses más relevantes, ya que representan el $20 \%$ de la precipitación total anual, serían febrero y octubre, con tendencias opuestas. Por su parte, un incremento del $130 \%$ de las precipitaciones de agosto no supondría una contribución notable a la evolución de las precipitaciones anuales ya que únicamente representan el $3 \%$ de la precipitación media total anual.

La evolución de las precipitaciones anuales (figura 5) integra las observaciones anteriormente expuestas y consecuentemente muestra una marcada variabilidad interanual y carece de tendencias significativas. En el gráfico se puede apreciar un ligero incremento desde el inicio del período hasta finales de la década de los 70, al que sigue un leve descenso hasta finales de los 90 y, finalmente, una suave recuperación en la última década. El test de MannMÓVIL DE 5 AÑOS APLICADAAL RANGO INTERCUARTIL (CUARTILES 1 Y 3).

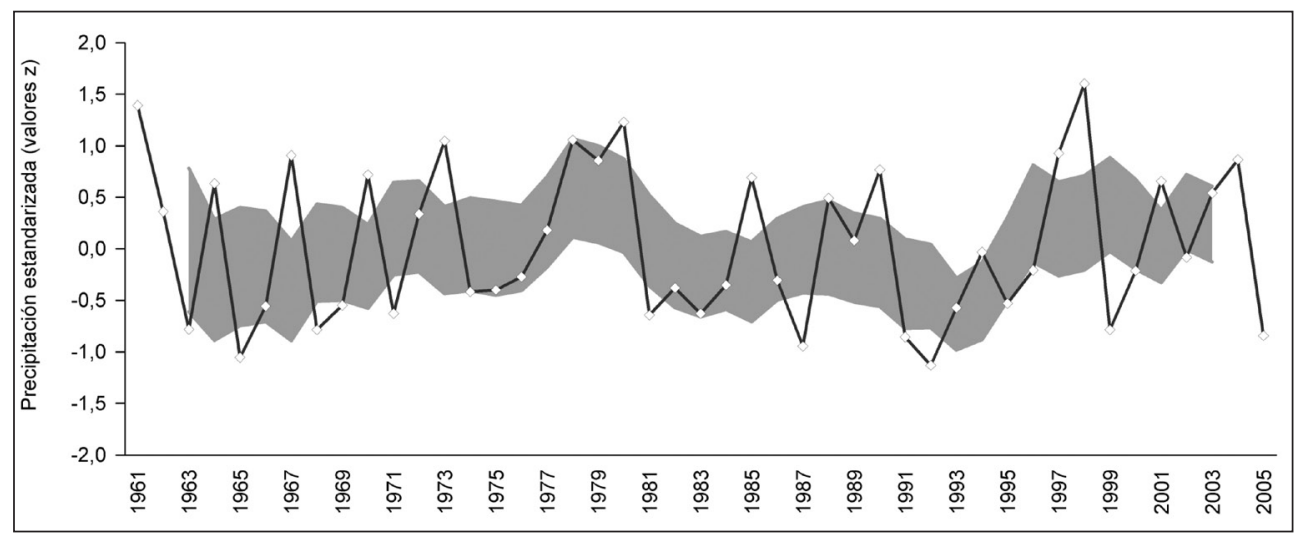


Figura 6

EVOLUCIÓN DE LAS PRECIPITACIONES MENSUALES DURANTE EL PERIODO DE ESTUDIO. LA LÍNEA CON ROMBOS REPRESENTA LOS VALORES DE PRECIPITACIÓN MEDIA DE LAS 214 ESTACIONES, EL SOMBREADO GRIS LA MEDIA MÓVIL DE 5 AÑOS APLICADA AL RANGO INTERCUARTIL (CUARTILES 1 Y 3)
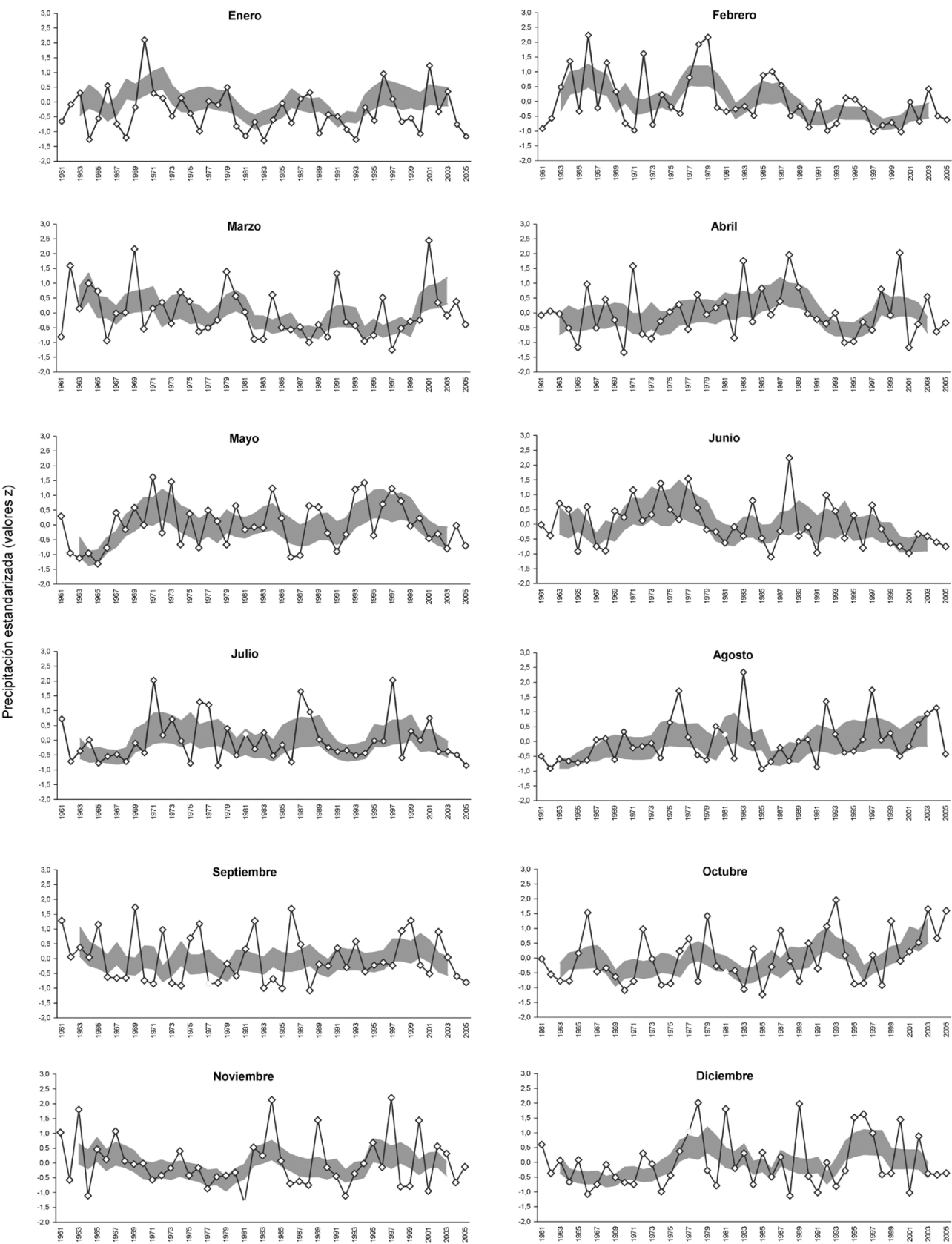
Tabla 2

ESTADÍSTICOS DE CENTRALIDAD, DISPERSIÓN Y CAMBIO PARA LAS PRECIPITACIONES MEDIAS DE LA CUENCA DEL DUERO DURANTE EL PERIODO 1961-2005. *: ESTADÍSTICAMENTE SIGNIFICATIVO CON UN 95\% DE CONFIANZA

\begin{tabular}{|l|c|c|c|c|c|c|c|}
\hline \multirow{2}{*}{ n= 214 } & \multirow{2}{*}{$\begin{array}{c}\text { Precipitación } \\
\text { media }(\mathbf{m m})\end{array}$} & $\begin{array}{c}\text { Contribución } \\
\text { al total anual } \\
\text { \% }\end{array}$ & $\begin{array}{c}\text { Coeficiente } \\
\text { de variación } \\
\text { medio \% }\end{array}$ & \multicolumn{2}{|c|}{ Ajuste lineal } & \multicolumn{2}{c|}{ Tendencia } \\
\cline { 5 - 8 } & & & $\mathbf{R}^{2}$ & Cambio \% & tau MK & $\boldsymbol{\alpha}$ \\
\hline Enero & 67,73 & 10,68 & 79,33 & 0,01 & $-13,89$ & $-0,09$ & 0,38 \\
\hline Febrero & 55,98 & 8,83 & 84,48 & 0,11 & $-56,41$ & $-0,18$ & 0,08 \\
\hline Marzo & 46,95 & 7,40 & 80,85 & 0,02 & $-24,53$ & $-0,10$ & 0,35 \\
\hline Abril & 57,85 & 9,12 & 65,47 & 0,00 & 8,93 & $-0,01$ & 0,92 \\
\hline Mayo & 62,86 & 9,91 & 59,48 & 0,02 & 18,97 & 0,08 & 0,45 \\
\hline Junio & 39,63 & 6,25 & 79,17 & 0,08 & $-24,00$ & $-0,23 *$ & 0,02 \\
\hline Julio & 21,67 & 3,42 & 112,43 & 0,00 & 4,55 & 0,01 & 0,89 \\
\hline Agosto & 19,81 & 3,12 & 108,27 & 0,09 & 133,33 & $0,24 *$ & 0,02 \\
\hline Septiembre & 40,55 & 6,39 & 83,86 & 0,00 & $-8,33$ & $-0,01$ & 0,95 \\
\hline Octubre & 68,31 & 10,77 & 75,58 & 0,11 & 104,44 & 0,20 & 0,05 \\
\hline Noviembre & 74,16 & 11,69 & 73,86 & 0,00 & $-7,79$ & $-0,07$ & 0,48 \\
\hline Diciembre & 70,97 & 11,19 & 82,07 & 0,02 & 43,10 & 0,07 & 0,51 \\
\hline AÑO & 634,29 & 100,00 & 23,33 & 0,00 & 0,16 & 0,04 & 0,72 \\
\hline
\end{tabular}

Figura 7

DISTRIBUCIÓN ESPACIAL DE LOS VALORES CORRESPONDIENTES AL COEFICIENTE DE VARIACIÓN DE LAS PRECIPITACIONES ANUALES DURANTE EL PERIOODO DE ESTUDIO

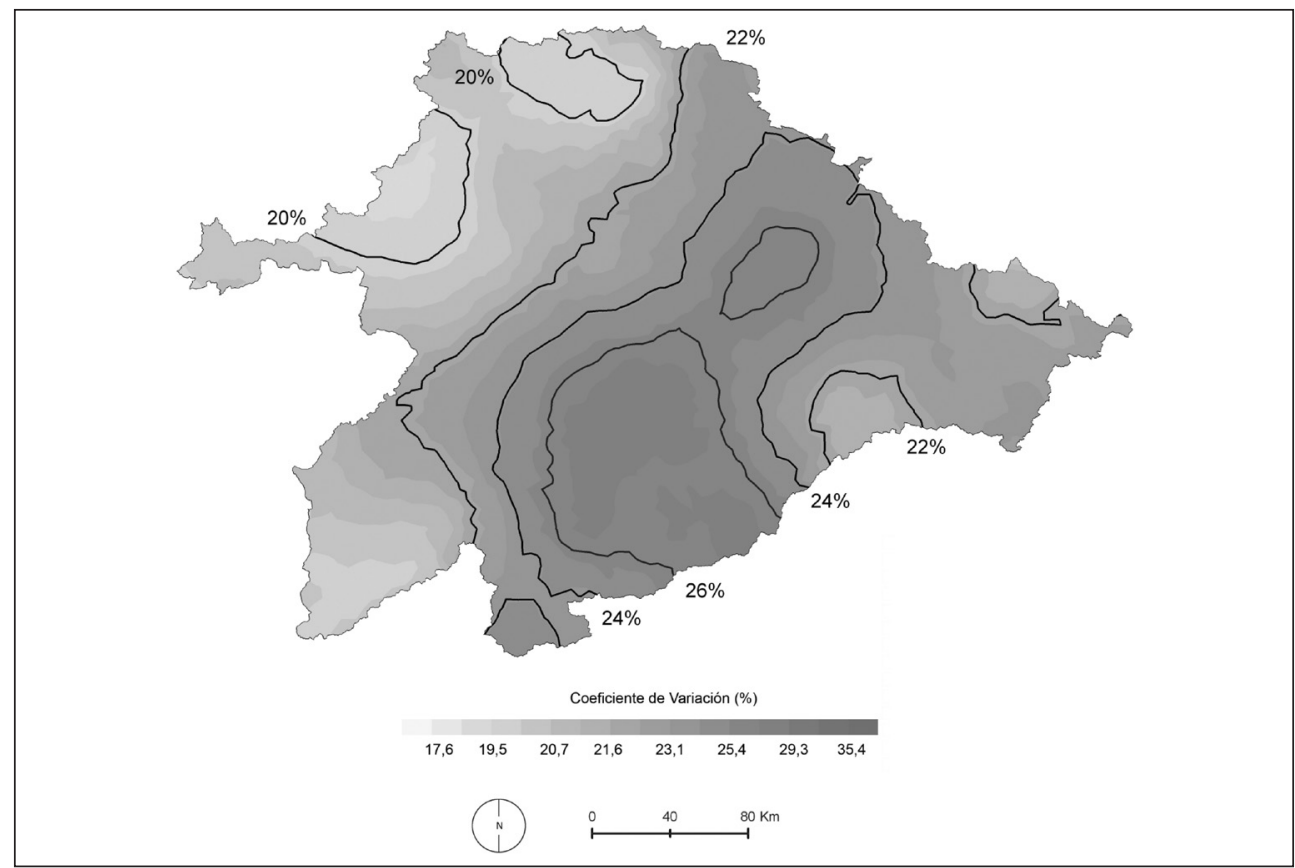


Figura 8

TENDENCIA DE LAS PRECIPITACIONES ANUALES Y MENSUALES EN LA CUENCA DEL DUERO DURANTE EL PERIOODO DE ESTUDIO. LOS TONOS OSCUROS Y CLAROS INDICAN TENDENCIAS POSITIVAS Y NEGATIVAS RESPECTIVAMENTE. LIINEAS NEGRA/BLANCA $\alpha<0,05$ (ESTADISTICAMENTE SIGNIFICATIVO CON UN $95 \%$ DE CONFIANZA). *: VALORES DEt SIGNIFICATIVOS AL $95 \%$

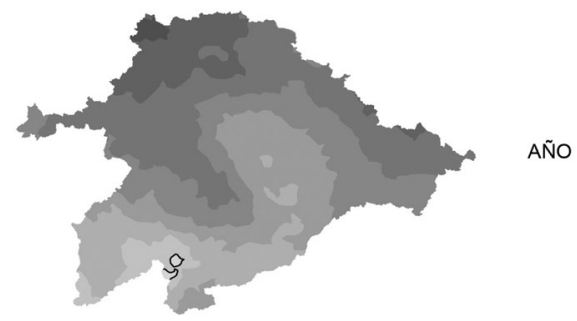

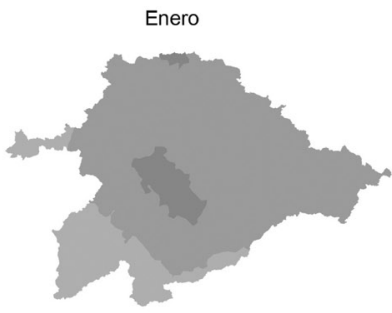

Abri
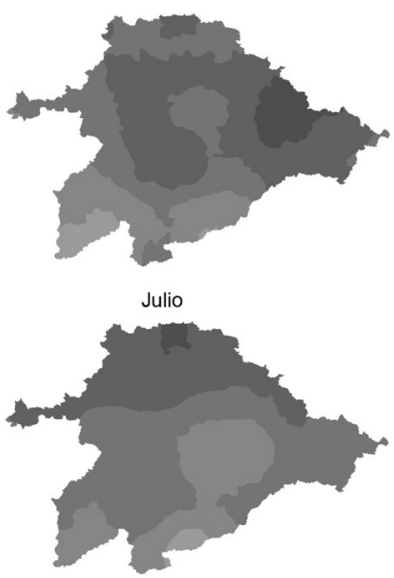

Octubre

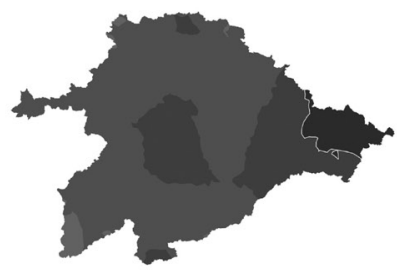

Febrero

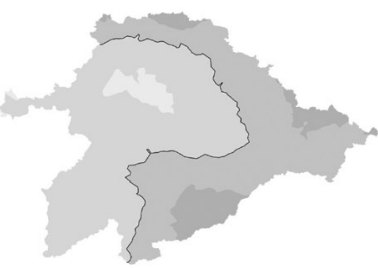

Mayo

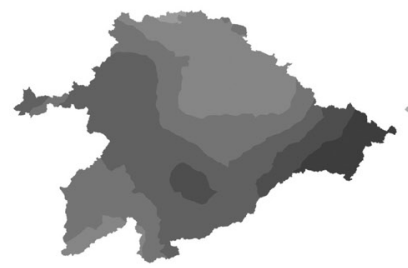

Agosto

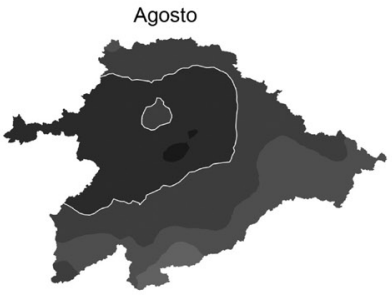

Noviembre

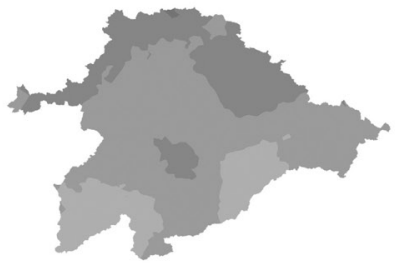

Tendencia (Tau de Mann-Kendall)

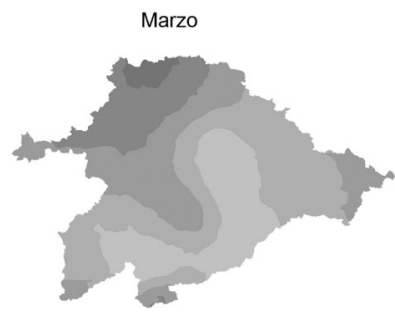

Junio
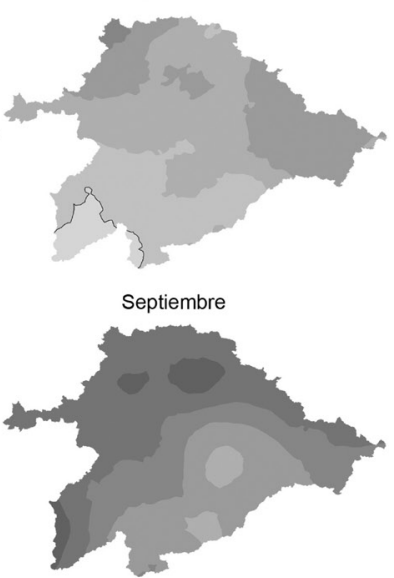

Diciembre

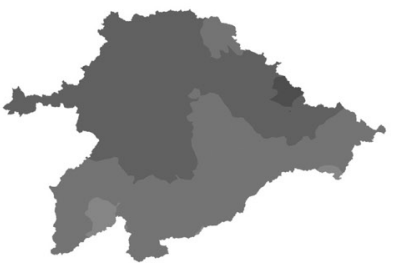

$-0,30$

$\begin{array}{lll}-0,20^{*} & -0,10 \quad 0,0\end{array}$

0,10

$0,20^{*}$

0,30 
Kendall aplicado a las precipitaciones anuales permite rechazar la existencia de una tendencia significativa para el periodo de estudio $(\tau=0,04 ; \alpha=0,72)$.

Por medio de la interpolación espacial de los valores $\tau$ de Mann-Kendall de cada de una de las estaciones pluviométricas se han obtenido los mapas que reflejan la distribución espacial de las tendencias tanto de las precipitaciones anuales como mensuales (figura 8). A pesar de que en el mapa correspondiente a las precipitaciones anuales se aprecia un patrón espacial en la distribución de las tendencias, con valores positivos en la mitad norte y valores negati-

Figura 9

TENDENCIA DE LAS PRECIPITACIONES EN LA CUENCA DEL DUERO. VARIABILIDAD ESPACIAL Y TEMPORAL: A) MENSUAL; B) ESTACIONAL YANUAL. DIAGRAMAS DE CAJA: LÍNEA NEGRA = MEDIANA, CAJA $=$ CUARTIL $1^{\circ} Y 3^{\circ}$ (RANGO INTERCUARTIL), BRAZOS = PERCENTILES 10 Y 90, PUNTOS = PERCENTILES 5 Y 95 DE LAS 214 SERIES DE PRECIPITACIÓN. LAS LÍNEAS GRISES DISCONTINUAS INDICAN EL UMBRAL DE SIGNIFICACIÓN ESTADÍSTICA $(\alpha<0,05)$
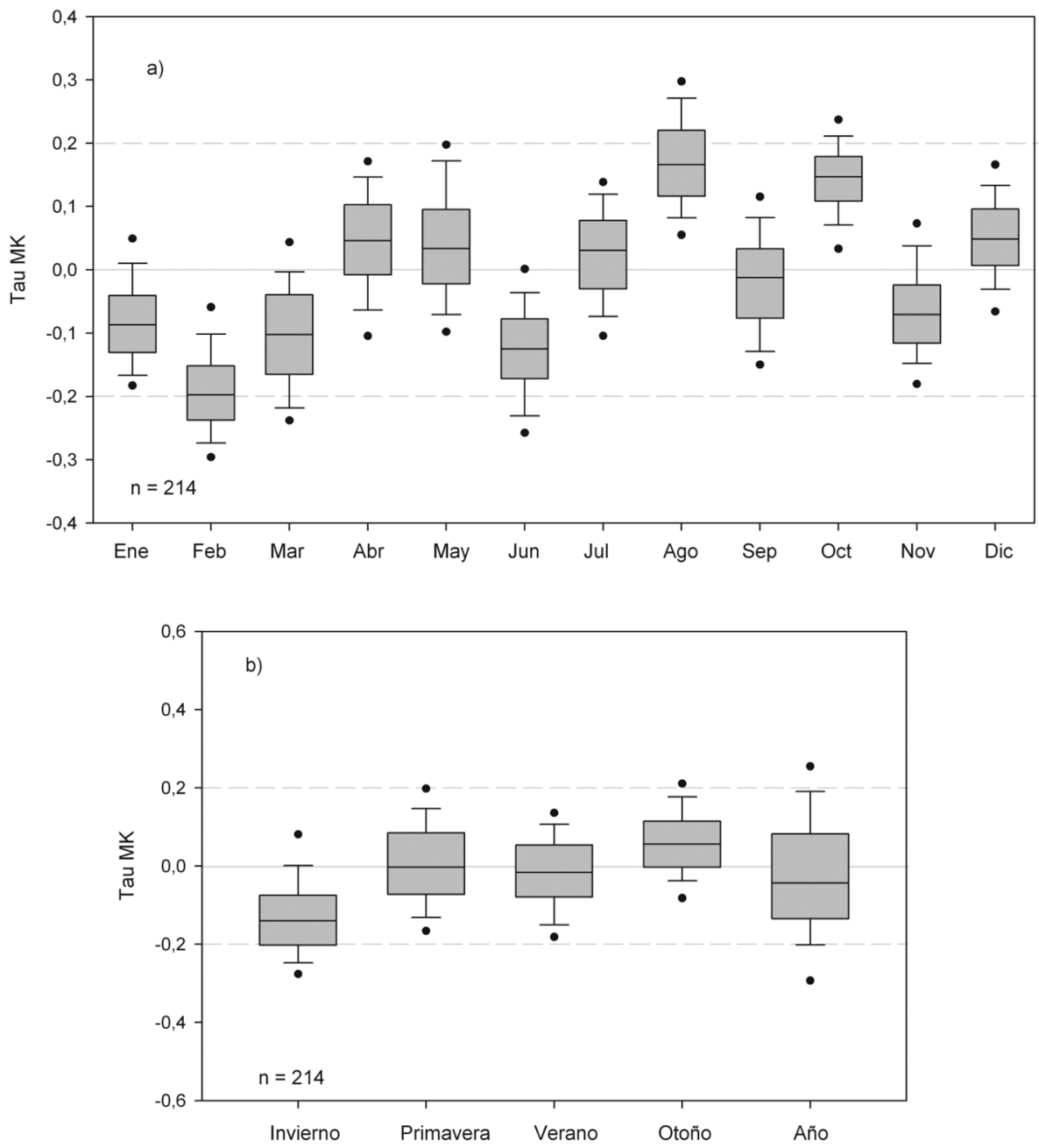
vos en la mitad sur, tan sólo en una pequeña zona del sector suroccidental las tendencias son estadísticamente significativas. De forma general, se observa que no existe ninguna homogeneidad en el signo de las tendencias a lo largo del año y que en la mayor parte de los casos los valores de las mismas no son significativos o sólo en una reducida parte de la cuenca. En los meses de enero, febrero, marzo y junio predominan los coeficientes negativos, aunque sólo son significativos en febrero en la mayor parte del territorio. Los meses de octubre, diciembre, abril, mayo, julio y agosto muestran tendencias positivas, pero únicamente en agosto estas son significativas en buena parte del territorio.

La figura 9 muestra una síntesis gráfica de las tendencias registradas para las precipitaciones mensuales (a) y estacionales y mensuales (b) de las 214 series pluviométricas. De forma general se observan coeficientes negativos en los meses de enero, febrero, marzo, junio, septiembre y octubre, aunque sólo en febrero el $50 \%$ de los casos (mediana) alcanza el umbral de la significación estadística. Por su parte, se registran coeficientes positivos en abril, mayo, julio, agosto, octubre y diciembre, aunque en la mayoría de los casos no son estadísticamente significativos. No se puede hablar de tendencias contrastadas, ya que los coeficientes, con independencia de su signo, no alcanzan el umbral de significación estadística. En la agregación estacional de las series (figura 9.b) se aprecia que sólo en invierno existe una ligera tendencia regresiva aunque sin ser estadísticamente significativa. Las series de precipitaciones anuales muestran indistintamente coeficientes positivos y negativos, constatándose que en el $90 \%$ de los casos no se alcanza el umbral de significación estadística.

Como se explicó con anterioridad, las precipitaciones en agosto son escasas y su aumento con el tiempo probablemente esté relacionado con un incremento de la frecuencia de tormentas convectivas. En los meses de enero, febrero, marzo y junio la evolución de las precipitaciones ha sido negativa pero sin significado estadístico con la excepción de una superficie importante de la cuenca durante el mes de febrero. Debido al control de la circulación general atmosférica sobre las precipitaciones invernales en la Península Ibérica, la disminución de las precipitaciones en esos meses muy probablemente esté controlada por el patrón de circulación que determina la llegada de los frentes atlánticos, es decir, la Oscilación del Atlántico Norte (NAO). Cuando el gradiente de presión entre el Anticiclón de las Azores y la Baja de Islandia es alto, la trayectoria de los frentes se desplaza hacia al norte provocando la ocurrencia de inviernos secos en el sur de Europa. Numerosos estudios (p. ej. Thompson et al., 2000; Osborn, 2004), muestran que esta situación ha sido frecuente en las últimas décadas lo que sugiere que esta haya sido la causa de la tendencia observada de las precipitaciones invernales en la cuenca del Duero.

\section{DISCUSIÓN Y CONCLUSIONES}

En términos generales los resultados obtenidos permiten afirmar que no existe una tendencia generalizada en las precipitaciones de la cuenca del Duero durante el período de estudio. Esta conclusión en cierto modo no se adecua a las previsiones publicadas en relación a la evolución de las precipitaciones durante el siglo XXI en un escenario de calentamiento global. En el caso del sur de Europa las predicciones son de un descenso general de las precipitaciones acompañado paradójicamente de una mayor frecuencia de eventos extremos (Trenberth et al. 2003, IPCC, 2007, Bladé y Castro-Diez, 2010). No obstante, estos mismos 
modelos apuntan que ese escenario será más probable en la mitad oriental de la Península Ibérica que en la occidental. Comparando nuestros resultados con los publicados por otros autores, que también han estudiado la evolución de las precipitaciones en distintos territorios del sur de Europa durante las últimas décadas, podemos comprobar que las diferencias entre los distintos estudios no son apreciables. Además, deberíamos considerar que los trabajos publicados comprenden diferentes períodos y áreas de estudio de extensión diversa, utilizan un número distinto de observatorios y también difieren en los tests estadísticos empleados (Giorgi, 2002, Norrant y Douguedroit, 2006).

Considerando los estudios realizados en distintos territorios de la Península Ibérica sobre la evolución de las precipitaciones durante las últimas décadas tampoco se ha podido constatar una tendencia clara y general en la evolución de las mismas durante la segunda mitad del siglo XX (p. ej. Serrano et al. 1999, De Castro et al. 2005). Los resultados que hemos obtenido en la cuenca del Duero se acercan al patrón general detectado a escala peninsular, aunque con algunos matices determinados por cuestiones metodológicas (período de estudio y número de observatorios). Por ejemplo, Paredes et al. (2006), utilizando 46 estaciones pluviométricas distribuidas por toda la Península, mostraron la existencia de un significativo descenso de las precipitaciones en el mes de marzo durante la segunda mitad del siglo XX en el oeste peninsular. En nuestro trabajo, con un número mucho mayor de observatorios, hemos observado que en marzo la tendencia también es regresiva, aunque no estadísticamente significativa, aunque sí lo es en febrero en buena parte del territorio. López-Bustins et al. (2008) analizaron un total de 51 series pluviométricas, también a escala peninsular, y detectaron tendencias negativas en los meses invernales de su sector occidental durante la segunda mitad del siglo pasado, mientras que en la parte oriental no observaron ninguna tendencia significativa. Estos autores además relacionan la dinámica observada de las precipitaciones con el comportamiento de diferentes teleconexiones vinculadas a la circulación general atmosférica: Oscilación Ártica, Oscilación del Atlántico Norte y Oscilación del Mediterráneo Occidental. Considerando un período de tiempo más largo (1880-1992), y los datos de las capitales de provincias españolas, Esteban-Parra et al. (1998) encontraron una tendencia decreciente de las precipitaciones totales anuales en los observatorios situados en el interior del territorio entre los 60 y los 90, siendo especialmente significativo en los meses primaverales. Recientemente González-Hidalgo et al. (2010) han publicado los resultados de un análisis para el conjunto de la Península Ibérica, utilizando 2.670 series homogeneizadas de precipitación correspondientes al período 1946-2005, concluyendo la enorme variabilidad espacial de las tendencias a escala mensual, siendo únicamente estas significativas, y con signo negativo, en el mes de marzo.

Esta variabilidad espacio-temporal de las precipitaciones también ha sido confirmada por trabajos realizados a mayor escala. Partiendo de un ámbito geográfico similar al de nuestro análisis, Labajo y Piorno (2001) no encontraron una tendencia general y significativa en las precipitaciones de Castilla y León para el período 1931-96, aunque sí apreciaron una tendencia decreciente entre 1972 y 1996. Del Río et al. (2005) analizaron 171 series pluviométricas en Castilla y León, considerando el período 1961-1997, y observaron un leve descenso, estadísticamente no significativo, en las series de precipitaciones anuales. En su análisis de las series mensuales detectaron tendencias negativas en febrero y marzo, siendo estadísticamente significativas en el último caso. En la Comunidad Valenciana De Luis et al. (2009) y 
González-Hidalgo et al. (2009) encontraron una acusada variabilidad espacio-temporal en sus análisis con tendencias, por ejemplo, decrecientes en las precipitaciones invernales en el interior y, sin embargo, positivas, en la misma estación, en la zona costera. En el caso del mes de marzo apreciaron una tendencia negativa casi general para el conjunto del territorio. El estudio de López-Moreno et al. (2010) obtuvo unos resultados similares a los anteriores en el caso del noreste de la Península Ibérica utilizando 217 series. En todos estos casos, fue posible determinar la variabilidad espacial de las series de precipitación gracias a la utilización de bases de datos con alta resolución espacial. Respecto a trabajos anteriores, realizados en el ámbito territorial de la cuenca del Duero, una de las principales aportaciones del presente artículo es haber contado con un número elevado de estaciones, lo cual ha permitido aplicar técnicas de interpolación espacial y observar con mayor precisión la variabilidad espacial en los resultados de los análisis.

La discrepancia entre las tendencias (o mejor dicho, la ausencia de tendencias) observadas durante el siglo XX y las estimaciones pronosticadas para las siguientes décadas del siglo XXI en la región mediterránea es sorprendente (García-Ruiz et al., 2011). El calentamiento de la región acaecido desde comienzos de los 70 hasta la actualidad teóricamente debería generar una mayor cantidad de vapor de agua, así como una redistribución del mismo en la atmósfera dando lugar a cambios apreciables en el ciclo hidrológico global (Milly et al. 2005, Held y Soden, 2006). Si a escala planetaria sí se ha detectado un incremento de las precipitaciones durante la segunda mitad del siglo XX, probablemente relacionado con el calentamiento global (Dai et al. 1997, New et al. 2001), en el caso de la región mediterránea la apreciable variabilidad espacio-temporal, característica de sus regímenes pluviométricos, explica que no se haya observado una tendencia clara con carácter general. A modo de conclusión, en nuestro caso hemos observado: i) Una apreciable variabilidad temporal, estacional y espacial de las precipitaciones en la cuenca del Duero. ii) Las tendencias temporales de las series mensuales y anuales, cuando existen, no son significativas desde un punto de vista estadístico. iii) La distribución espacial de las tendencias de las precipitaciones anuales muestra un cierto contraste entre las tendencias positivas observadas en la mitad norte de la cuenca y las negativas de la mitad sur. iv) Sólo en el mes de febrero se ha detectado una tendencia negativa y significativa de las precipitaciones en más de la mitad del territorio del sector español de la cuenca del Duero. De momento este escenario no se corresponde con la previsión de una reducción de las precipitaciones, estimada en un 7\%, para el año 2060 (Ayala-Carcedo e Iglesias, 2000) lo que sugiere a corto plazo continuar con este tipo de análisis.

A modo de apéndice, y con carácter aplicado, queremos finalizar nuestro trabajo con la siguiente reflexión: sin duda las precipitaciones son el origen de los recursos hídricos de un territorio, debido a lo cual es imprescindible considerar cualquier cambio en las mismas, a medio y largo plazo, en relación con la disponibilidad de agua en una cuenca concreta. En el caso de la región mediterránea, donde se encuentra emplazada la cuenca del Duero, diversos estudios pronostican una reducción de sus recursos hídricos cercana al $20 \%$ (Milly et al., 2005; IPCC, 2007). Estas predicciones sobre la disponibilidad de los recursos hídricos se centran en la evolución futura de las precipitaciones como variable predictora. Nuestro trabajo muestra que las precipitaciones en la cuenca del Duero no han variado apreciablemente a medio plazo, aunque se perciben sutiles cambios estacionales relacionados con las tenden- 
cias correspondientes a los meses de febrero, agosto y octubre. Como consecuencia de ello podemos esperar en un futuro inmediato pequeñas variaciones en los regímenes fluviales de la cuenca, pero sin llegar al descenso neto de los recursos hídricos que aventuran las predicciones. Sin embargo, diversos estudios llevados a cabo sobre la evolución de los recursos hídricos en la propia cuenca del Duero han demostrado un importante descenso en el caudal de los ríos, tanto en los tramos de cabecera como en los cursos bajos, acompañado de marcados cambios estacionales (Gallart y Llorens, 2002; Ceballos et al., 2008; Morán-Tejeda et al., 2011a). En otros territorios de la Península Ibérica, como la cuenca del Ebro, también se ha comprobado una evolución contrastada entre precipitaciones y caudales, demostrándose que el incremento de la cubierta vegetal en las cuencas de cabecera ha sido el factor responsable del descenso de los recursos hídricos ante unas precipitaciones que no han descendido de forma apreciable (Beguería et al., 2003; López-Moreno et al., 2011; Morán-Tejeda et al., 2011b). Estas observaciones remarcan el interés por seguir analizando la evolución futura de las precipitaciones desde una perspectiva crítica, ya que el descenso pluviométrico puede ir acompañado de un aumento de la evapotranspiración debido al aumento de la cubierta vegetal y el incremento térmico, produciéndose con ello un retroceso aún más acusado de los recursos hídricos en las cuencas mediterráneas.

\section{AGRADECIMIENTOS}

El presente estudio ha sido posible gracias a la financiación recibida de la Junta de Castilla y León (proyecto SA099A06) y al disfrute, por parte del primer autor, de una beca predoctoral de investigación concedida por la Junta de Castilla y León (EDU/918/20069). Los autores también queremos expresar nuestro sincero agradecimiento a los dos revisores anónimos del trabajo original por sus comentarios y sugerencias.

\section{BIBLIOGRAFÍA}

ALEXANDERSSON, H. (1986): «A Homogeneity test applied to precipitation data». Journal of Climatology, $\mathrm{n}^{\circ}$ 6, 661-675.

AYALA-CARCEDO, F.J., IGLESIAS, A. (2000): «Impactos del posible cambio climático sobre los recursos hídricos, el diseño y la planificación hidrológica en la España peninsular». El Campo de las Ciencias y las Artes, n 137, 201-222.

BEGUERÍA, S., LÓPEZ-MORENO, J. I., LORENTE, A., SEEGER, M. y GARCÍA-RUIZ. J.M. (2003): «Assessing the effect of climate oscillations and land-use changes on streamflow in the Central Spanish Pyrenees». Ambio n ${ }^{\circ} 32,283-286$.

BERRYMAN, D., BOBEE, B., CLUIS, D. y HAEMMERLI, J. (1988): «Nonparametric tests for trend detection in water-quality time series». Water Resources Bulletin, $\mathrm{n}^{\circ} 24$, 545-556.

BLADÉ, I. y CASTRO DÍEZ, Y. (2010): «Tendencias atmosféricas en la Península Ibérica durante el periodo instrumental en el contexto de la variabilidad natural». En Clima en España: pasado, presente y futuro (Pérez, F. y Boscolo, R., Eds). Madrid, Red Temática CLIVAR-España, 25-42. 
CABO, A. y MANERO, F. (1990): «Castilla y León». En Geografía de España (Bosque Maurel, J. y Vilá Valentí, J. -Eds.-). Barcelona, Planeta, 255-591.

CEBALlOS BARBANCHO, A., MORÁN TEJEDA, E., LUENGO UGIDOS, M.A., y LLORENTE-PINTO, J.M. (2008): «Water resources and environmental change in a Mediterranean environment: The south-west sector of the Duero river basin (Spain)». Journal of Hydrology, $\mathrm{n}^{\circ} 351,126-138$.

DAI, A., FUNG, I.Y. y DELGENIO, A. D. (1997): «Surface observed global land precipitation variations during 1900-88». Journal of Climate, $\mathrm{n}^{\circ}$ 10, 2943-2962.

DE CASTRO, M., MARTÍN VIDE, J. y ALONSO, S. (2005): «El clima de España: pasado, presente y escenarios de clima para el siglo XXI». En Evaluación preliminar de los impactos en España por efecto del cambio climático (Moreno Rodríguez, J. M. C. Ed). Ministerio de Medio Ambiente y Universidad de Castilla-La Mancha, 1-64.

DE LUIS, M., GONZÁLEZ HIDALGO, J. C., LONGARES, L. A. y STEPANEK, P. (2009): «Seasonal precipitation trends in the Mediterranean Iberian Peninsula in second half of 20th century». International Journal of Climatology, $\mathrm{n}^{\circ}$ 29, 1312-1323.

DEL RÍO, S., PENAS, A. y FRAILE, R. (2005): «Analysis of recent climatic variations in Castile and Leon (Spain)». Atmospheric Research, $\mathrm{n}^{\circ}$ 73, 69-85.

EASTERLING, D. R. y PETERSON, T. C. (1995): «The effect of artificial discontinuities on recent trends in minimum and maximum temperatures». Atmospheric Research, $\mathrm{n}^{\circ} 37$, 19-26.

ESTEBAN PARRA, M. J., RODRIGO, F. S. y CASTRO DIEZ, Y. (1998): «Spatial and temporal patterns of precipitation in Spain for the period 1880-1992». International Journal of Climatology, $\mathrm{n}^{\circ} 18,1557-1574$.

FERNÁNDEZ, J. (Ed.) (1992). Análisis del Medio Físico de Castilla y León. Síntesis regional. Valladolid. Junta de Castilla y León.

GALLART, F. y LLORENS, P. (2002): «Water resources and environmental change in Spain. A key issue for sustainable catchment management». En Environmental Change and Water Sustainability (García Ruiz, J.M., Jones, J.A.A. y Arnaez, J. -Eds.-). Zaragoza, IPE-CSIC, 11-20.

GARCÍA FERNÁNDEZ, J. (1986): El clima en Castilla y León. Valladolid. Ámbito.

GARCÍA RUIZ, J.M., LÓPEZ MORENO, J.I., VICENTE SERRANO. S., LASANTA MARTÍNEZ, T. y BEGUERÍA, S. (2011): «Mediterranean water resources in a global change scenario». Earth-Science Review, no 105, 121-139.

GIORGI, F. (2002): «Variability and trends of sub-continental scale surface climate in the twentieth century. Part I: observations». Climate Dynamics, ${ }^{\circ}$ 18, 675-691.

GONZÁLEZ HIDALGO, J.C., BRUNETTI, M. y DE LUIS, M. (2010): «A new tool for monthly precipitation analysis in Spain: MOPREDAS database (monthly precipitation trends December 1945-November 2005)». International Journal of Climatology, $\mathrm{n}^{\circ} 31$, 715-731.

GONZALEZ HIDALGO, J.C., LOPEZ-BUSTINS, J.A., STEPANEK, P., MARTIN VIDE, J. y DE LUISA, M. (2009): «Monthly precipitation trends on the Mediterranean fringe of the Iberian Peninsula during the second-half of the twentieth century (1951-2000)». International Journal of Climatology, $\mathrm{n}^{\circ}$ 29, 1415-1429. 
HELD, I.M. y SODEN, B.J. (2006): «Robust responses of the hydrological cycle to global warming». Journal of Climate, $\mathrm{n}^{\circ} 19,5686-5699$.

HIRSCH, R. M., SLACK, J. R. y SMITH, R. A. (1982): «Techniques of trend analysis for monthly water quality data». Water Resour. Res. 18:107-121.

IPCC (2007): Climate Change 2007: Synthesis Report. [Core Writing Team, Pachauri RK and Reisinger A (Eds.)]. IPCC: Geneva, Switzerland, 104 pp. Contribution of Working Groups I, II and III to the Fourth Assessment Report of the Intergovernmental Panel on Climate Change.

JIMÉNEZ, S. (1997): Mapa Geológico y Minero de Castilla y León, Escala 1/400.000. Valladolid. SIEMCALSA.

KEISER, D.T. y GRIFFITHS, J.F. (1997): «Problems associated with homogeneity testing in climate variation studies: A case study of temperature in the northern Great Plains, USA». International Journal of Climatology, $\mathrm{n}^{\circ}$ 17, 497-510.

KENDALL, M.G. (1975): Kendall Rank Correlation Methods. London. Griffin.

LABAJO, J.L. y PIORNO, A. (2001): «Regionalisation of precipitation in Castilla and Leon (Spain). Analysis of its temporal behaviour». En Detecting and Modelling Regional Climate Change (Brunet, M. y López-Bonillo, D., Eds). Springer, 163-173.

LANZANTE, J.R. (1996): «Resistant, robust and non-parametric techniques for the analysis of climate data: Theory and examples, including applications to historical radiosonde station data». International Journal of Climatology, $\mathrm{n}^{\circ}$ 16, 1197-1226.

LOPEZ-BUSTINS, J.A., MARTIN VIDE, J. y SANCHEZ LORENZO, A. (2008): «Iberia winter rainfall trends based upon changes in teleconnection and circulation patterns». Global and Planetary Change, $\mathrm{n}^{\circ}$ 63, 171-176.

LÓPEZ MORENO, J.I., VICENTE SERRANO, S.M., ANGULO MARTÍNEZ, M., BEGUERÍA, S. y KENAWY,A. (2010): «Trends in daily precipitation on the northeastern Iberian Peninsula, 1955-2006». International Journal of Climatology, $\mathrm{n}^{\circ}$ 30, 1026-1041.

LÓPEZ MORENO, J.I., VICENTE SERRANO, S.M. MORÁN TEJEDA, E. ZABALZA, J. LORENZO LACRUZ, J. y GARCÍA RUIZ. J.M. (2011): «Impact of climate evolution and land use changes on water yield in the Ebro basin». Hydrology and Earth System Sciences $\mathrm{n}^{\circ} 15,311-322$.

MANN, H. B. (1945): «Non parametric test against trend». Econometrica, n 13, 245-259.

MILLY, P.C.D., DUNNE, K.A. y VECCHIA, A.V. (2005): «Global pattern of trends in streamflow and water availability in a changing climate». Nature, $\mathrm{n}^{\circ} 438,347-350$.

MOBERG, A. y BERGSTROM, H. (1997): «Homogenization of Swedish temperature data .3. The long temperature records from Uppsala and Stockholm». International Journal of Climatology, n ${ }^{\circ} 17,667-699$.

MORÁN TEJEDA, E., CEBALLOS BARBANCHO, A. LLORENTE PINTO, J. M. y LÓPEZ MORENO, J. I. (2011a): «Land-cover changes and recent hydrological evolution in the Duero Basin (Spain)». Regional Environmental Change: 1-17. Doi:10.1007/ s10113-011-0236-7.

MORÁN TEJEDA, E., LÓPEZ MORENO, J. I., CEBALLOS BARBANCHO, A. y VICENTE SERRANO. S. M. (2011b): «River regimes and recent hydrological changes in the Duero Basin (Spain)». Journal of Hydrology. Doi:10.1016/j.jhydrol.2011.04.034. 
NEW, M., TODD, M., HULME, M. y JONES, P. (2001): «Precipitation measurements and trends in the twentieth century». International Journal of Climatology, $\mathrm{n}^{\circ} 21,1899-1922$.

NORRANT, C. y DOUGUEDROIT, A. (2006): «Monthly and daily precipitation trends in the Mediterranean (1950-2000)». Theoretical and Applied Climatology, $\mathrm{n}^{\circ}$ 83, 89-106.

NINYEROLA, M., PONS, X. y ROURE, J.M. (2000): «A methodological approach of climatological modelling of air temperature and precipitation through GIS techniques». International Journal of Climatology, $\mathrm{n}^{\circ}$ 20, 1823-1841.

OSBORN, T. J. (2004): «Simulating the winter North Atlantic Oscillation: the roles of internal variability and greenhouse gas forcing». Climate Dynamics, $\mathrm{n}^{\circ} 22,605-623$.

PAREDES, D., TRIGO, R.M., GARCIA HERRERA, R. y TRIGO, I.F. (2006): «Understanding precipitation changes in Iberia in early spring: Weather typing and storm-tracking approaches». Journal of Hydrometeorology, $\mathrm{n}^{\circ}$ 7, 101-113.

PETERSON, T.C. y EASTERLING, D.R. (1994): «Creation of homogeneous composite climatological reference series». International Journal of Climatology, $\mathrm{n}^{\circ}$ 14, 671-679.

PETERSON, T.C., EASTERLING, D.R., KARL, T.R., GROISMAN, P., NICHOLLS, N., PLUMMER, N., TOROK, S., AUER, I., BOEHM, R., GULLETT, D., VINCENT, L., HEINO, R., TUOMENVIRTA, H., MESTRE, O., SZENTIMREY, T., SALINGER, J., FORLAND, E.J., HANSSEN BAUER, I., ALEXANDERSSON, H., JONES, P. y PARKER, D. (1998): «Homogeneity adjustments of in situ atmospheric climate data: A review». International Journal of Climatology, $\mathrm{n}^{\circ}$ 18, 1493-1517.

RODRÍGUEZ PUEBLA, C., ENCINAS, A. H. y SÁENZ, J. (2001): «Winter precipitation over the Iberian peninsula and its relationship to circulation indices». Hydrology and Earth System Sciences, $\mathrm{n}^{\circ}$ 5, 233-244.

RUIZ DE LA TORRE, J. R. (1996): Mapa Forestal de España. Madrid. Ministerio de Agricultura, Pesca y Alimentación. Dirección General de Conservación de la Naturaleza.

SERRANO, A., MATEOS, V.L. y GARCIA, J.A. (1999): «Trend analysis of monthly precipitation over the Iberian Peninsula for the period 1921-1995». Physics and Chemistry of the Earth Part B Hydrology Oceans and Atmosphere, $\mathrm{n}^{\circ} 24,85-90$.

SNEYERS, R. (1990): On the statistical analysis of series of observations. Geneva. Technical Note - World Meteorological Organization,.

SNEYERS, R. (1992): «Use and misuse of statistical methods for detection of climate change». Climate Change Detection Project, Report on the Informal Planning Meeting on Statistical Procedures for Climate Change Detection. WCDMP.

TABACHNICK, B. y FIDELL, L. (1996): «Using multivariate statistics». Third edition. New York. Harper Collins.

THOMPSON, D.W., WALLACE, J.M. y HEGERL, G.C. (2000): «Annular modes in the extratropical circulation. Part II: Trends». Journal of Climate, $\mathrm{n}^{\circ}$ 13, 1.018-1.036.

TRENBERTH, K.E., DAI, A., RASMUSSEN, R.M. y PARSONS, D.B. (2003): «The changing character of precipitation». Bulletin of the American Meteorological Society, $\mathrm{n}^{\circ} 84$, 1.205-1.217.

TRIGO, R.M. y PALUTIKOF, J.P. (2001): «Precipitation scenarios over Iberia: A comparison between direct GCM output and different downscaling techniques». Journal of Climate, $\mathrm{n}^{\mathrm{o}} 14,4.422-4.446$. 
VICENTE SERRANO, S.M., BEGUERÍA, S., LÓPEZ MORENO, J. I., GARCÍA VERA, M.A. y STEPANEK, P. (2010): «A complete daily precipitation database for northeast Spain: reconstruction, quality control, and homogeneity». International Journal of Climatology, $\mathrm{n}^{\mathrm{o}} 30,1.146-1.163$.

VISBECK, M.H., HURRELL, J.W., POLVANI, L. y CULLEN, H.M. (2001): «The North Atlantic Oscillation: Past, present, and future». Proceedings of the National Academy of Sciences of the United States of America, $\mathrm{n}^{\circ}$ 98, 12.876-12.877.

YUE, S., PILON, P. y CAVADIAS, G. (2002): «Power of the Mann-Kendall and Spearman's rho tests for detecting monotonic trends in hydrological series». Journal of Hydrology, $\mathrm{n}^{\mathrm{o}} 259,254-271$.

ZHANG, X.B., ZWIERS, F.W., HEGERL, G.C., LAMBERT, F.H., GILLETT, N.P., SOLOMON, S., STOTT, P.A. y NOZAWA, T. (2007): «Detection of human influence on twentieth-century precipitation trends». Nature, $\mathrm{n}^{\circ} 448,461-464$.

\section{ENLACES DE INTERNET:}

www.chduero.es

www.hercules.cedex.es 
